\title{
PROTON RADIOGRAPHY OF EXPLOSIVELY DISPERSED METAL PARTICLES WITH VARYING CARRIER FLUID
}

\author{
KYLE HUGHES ${ }^{1}$, KATHY PRESTRIDGE ${ }^{1}$, NAM-HO KIM ${ }^{2}$, \\ RAPHAEL HAFTKA ${ }^{2} \&$ SIVARAMAKRISHNAN BALACHANDAR ${ }^{2}$ \\ ${ }^{1}$ Physics Division, Los Alamos National Laboratory, USA \\ ${ }^{2}$ Department of Mechanical and Aerospace Engineering, University of Florida, USA
}

\begin{abstract}
A series of experiments performed at Los Alamos National Laboratory aimed to provide validation data for numerical simulations performed at the Centre of Compressible Multiphase Turbulence at the University of Florida. Five explosive tests were performed. Approximately $2.8 \mathrm{~g}$ of PBX-9501 was initiated by a RP- 80 detonator. A 13 × $6 \mathrm{~mm}$ cylindrical packet of $100 \mu \mathrm{m}$ steel particles was dispersed by the explosive. Three ambient carrier fluids were investigated: air, xenon, and $\mathrm{SF}_{6}$. To penetrate the optically opaque explosive products at the early time and track the dispersed particle cloud, radiography was required. Proton radiography performed at the Los Alamos Neutron Science Centre allowed extraction of 21 radiograph images at $2 \mu \mathrm{s}$ temporal resolution. Upstream and downstream particle fronts were extracted from the transmission radiographs. Centreline particle fronts for the three carrier phases demonstrated close agreement, suggesting the shock traversing the bed of particles provided little additional impulse to the dispersed particles in this regime. An additional shot was performed in vacuum and showed close agreement with the carrier phase shots, furthering this conclusion.

Keywords: multiphase flow, particle bed, explosive dispersal, proton radiography.
\end{abstract}

\section{INTRODUCTION}

The experiments considered in this investigation are to demonstrate the predictive ability of the code developed by the Centre of Compressible Multiphase Turbulence (CCMT) at the University of Florida. Compressible multiphase turbulent flows are a challenging and rich topic within the fluid mechanics community. Such a flow may arise in the natural world in the case of dispersal of particulate from a volcanic eruption or in engineering applications such as dispersal of particles by ordinance. Large-scale, exploratory experiments have been performed by Frost and collaborators [1]-[4] examining the behaviour of a bed of particles subjected to a detonation wave. The detonation replaces the central explosive with a highdensity, high-temperature gas that propagates outward. A shock wave travels through the particle bed, followed by a contact discontinuity between the shocked air and the explosive products. In the early times, the detonation wave causes many of the particles to collide and compact with each other. As the particles disperse, the experiments observe a series of largescale jets or fingers that form in the advancing particle front.

Small scale experiments by Sun, Tanno, and collaborators [5]-[7] have measured the forces on a single, stationary particle subjected to a moderate shock. When comparing the experimental results to drag relations proposed as compressible generalizations of the Maxey-Riley-Gatignol equation [8], [9], the generalized force formula captures the magnitude and the timing of the peak force quite well [10]-[12]. Shock-tube experiments examining the behaviour of moving particles have been performed by variety of researchers [13]-[16]. In these experiments, the position of the particle is observed after being subjected to a shock and the time-varying drag force extracted. Together these small scale experiments provide a wealth of validation data in the modest Mach number regime for a single particle for the proposed drag force relations. Hughes et al. [17] attempted to extend the validation 
data for a single particle, where the particles are subjected both to a high Mach number shock and a strong contact discontinuity.

Similar to the investigations of a single particle, other investigations have been performed at where many particles are subjected to a moderate Mach number shock. In this regime, not only are the individual drag forces important, but the particles compact and collide with each other as well. However, the flows still lack the strong contact discontinuity present in detonation conditions. Dilute suspensions of particles $(\varphi<1 \%)$ subjected to a shock have been studied extensively by researchers such as Rudinger [18]. Less common is examination of multiphase flows where moderate to large volume fractions are present $(\varphi<5 \%)$. Wagner et al. [19] examined shock wave impingement on a free-falling particle curtain of approximately $20 \%$ volume fraction by tracking the upstream and downstream particle fronts through high-speed Schlieren photography. Under very similar experimental conditions, Kellenberger et al. [20] examined the behaviour of stationary particle wafers of approximately $48 \%$ volume fraction subjected to a shock wave through high-speed photography. Note in these studies the volume fraction is less than the $60 \%$ commonly found in particle beds typical for the explosive dispersal of particles [21].

Examining the current level of experiments present in the literature, it was desirable to produce a set of experiments that contained all the physics of the large-scale explosive tests, but much reduced in the number of particles. The large-scale experiments contain $\mathrm{O}\left(10^{7}-\right.$ $\left.10^{9}\right)$ particles, whereas the new experiments are estimated to contain $\mathrm{O}\left(10^{5}\right)$ particles. The reduction of particles is a significant reduction in computational burden while still providing validation data within the high volume fraction and high Mach number flow.

\section{EXPERIMENTAL SETUP}

The experiments were performed at the Los Alamos Neutron Science Centre Proton Radiography facility. The proton radiography apparatus utilizes $800 \mathrm{MeV}$ protons provided by a linear particle accelerator [22]. A brief schematic is shown in Fig. 1. A beam of collimated protons is passed through the object of interest. The protons are guided by a series of magnetic lenses to focus the protons on the image plane, where a scintillator converts the protons to photons. The photons are turned out of the beam path with a mirror and passed to the camera group.

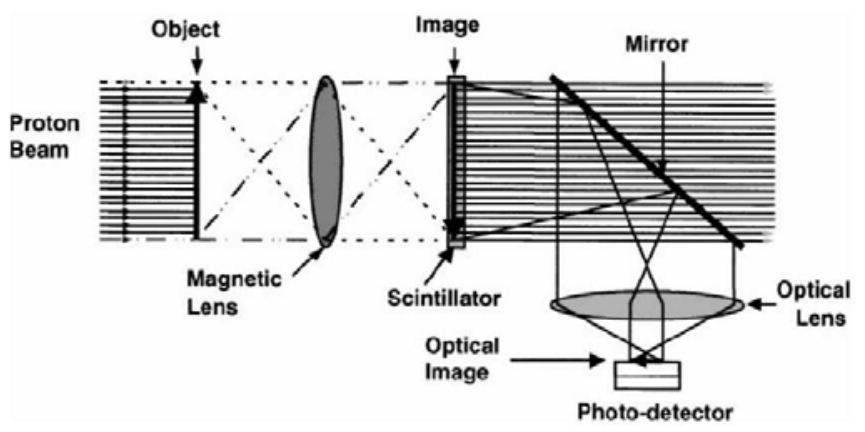

Figure 1: Proton radiography schematic showing the collimated beam of protons, optical system to capture the radiographs, and scintillator [22].

The protons pierce the opaque explosive products produced during detonation to provide 21 time-resolved images of the particle fronts. Images are spaced $2 \mu$ s apart with a resolution of $844 \times 853$ pixels. The $x 3$ magnifier is used to provide a $45 \mathrm{~mm}$ by $45 \mathrm{~mm}$ field of view with 
a resolution of $50 \mu \mathrm{m} /$ pixel. Tests are performed within a $1.83 \mathrm{~m}$ diameter spherical containment vessel that is pumped down to vacuum.

Details of the test article are shown in Fig. 2. A $10.2 \mathrm{~cm}$ outer diameter steel casing (AISI 4340) was used with a central bore of $13.1 \mathrm{~mm}$ nominal diameter and a depth of $19.1 \mathrm{~mm}$. The casing showed minor deformation post-test, but no fragmentation was observed. Examination of post-detonation photographs shows the bore diameter increased from 13.1 $\mathrm{mm}$ to $15 \pm 3 \mathrm{~mm}$, an increase of approximately 15\%. A pellet of PBX-9501 (12.7 mm diameter with $12.7 \mathrm{~mm}$ length) is initiated by a Teledyne RISI RP-80 exploding bridgewire detonator. When examining Fig. 2 a slight step is visible along the length of the centre bore. The step was necessary to locate the pellet of PBX-9501 exactly and provide a consistent particle bed depth. The PBX-9501 pellet was epoxied to this step using Barcobond to prevent movement of the explosive as the detonator is brought into contact with the explosive using the adapter nut. Time is measured from detonator initiation, determined from a current viewing resister, with approximately $\pm 0.1 \mu$ s uncertainty.

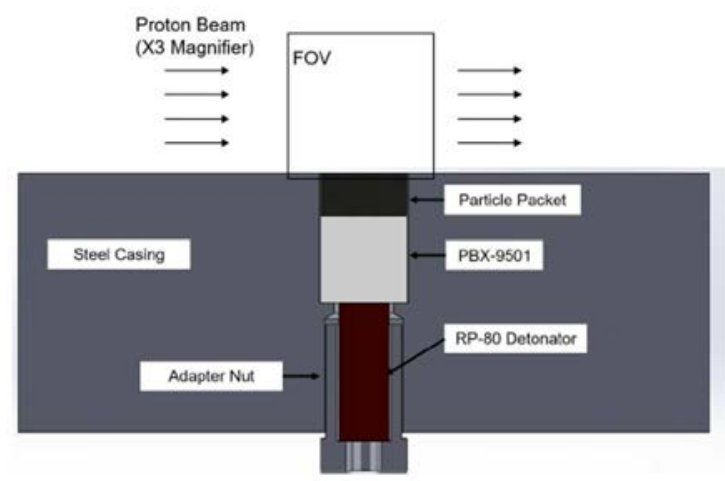

Figure 2: Cross-sectional view showing the particle packet and explosive train. Note the small step used to locate the pellet of PBX-9501.

After setting the explosive, a $13.1 \mathrm{~mm}$ diameter pocket with a depth of $6.35 \mathrm{~mm}$ was then filled with a steel particle packet and covered with tape. The tape was necessary to secure the particle bed during loading of the test article into the test vessel due to the significant jostling present in the loading process. The steel particles were 410 alloy, sieved between 75-125 $\mu \mathrm{m}$, and obtained from Sandvik Osprey. The steel particles were divided into three layers of $1.233 \pm 0.001 \mathrm{~g}$ by paper dividers $(3.697 \pm 0.002 \mathrm{~g}$ total). The particles were packed to approximately $56 \pm 1 \%$ volume fraction and no binder was used to constrain their movement.

The test articles were placed in the $1.83 \mathrm{~m}$ diameter pressure vessel, oriented vertically so the particles were held in place by gravity before detonation as well as by the tape. Wrapping the test article was a $6.35 \mathrm{~mm}$ thick aluminium cylinder to contain the various carrier phases. A gas handling system was used to ensure each cylinder was brought to $100 \pm 2 \mathrm{kPa}$ pressure and the temperature recorded to determine the initial thermodynamic state of the gas. Xenon and $\mathrm{SF}_{6}$ were chosen for their large nuclear cross-sections in attempt to capture the shock with the proton radiography. A series of traverses were used to align the centre of the test article with the proton beam. Table 1 contains a shot log detailing the gas used in each shot and the measured pressure and temperature. 
Table 1: Carrier fluid used in each shot with measured temperature and pressure.

\begin{tabular}{|c|c|c|c|}
\hline Shot \# & Gas & $\begin{array}{c}\text { Temperature, } \\
\mathrm{K}\end{array}$ & $\begin{array}{c}\text { Pressure, } \\
\mathrm{kPa}\end{array}$ \\
\hline 1 & Air & 298.6 & 101.67 \\
\hline 2 & Xenon & 297.3 & 100.13 \\
\hline 3 & SF $_{6}$ & 298.5 & 100.22 \\
\hline 4 & Air & 299.2 & 100.32 \\
\hline 5 & - & - & - \\
\hline
\end{tabular}

\section{RESULTS}

Sample transmission radiographs of the four different test conditions $40 \mu \mathrm{s}$ after detonation initiation are shown in Fig. 3. Note that an aluminium ring was placed around the lip of the steel casing to secure it and is visible in the radiographs as a thin layer of less dense material above the dark steel casing. Two screws used to secure the restraining ring are also visible at the edges of the radiographs. The particle packet shows a piston-like behaviour as it is expelled by the explosive. The three initial layers maintain their structure as they are expelled from the barrel and exhibit minimal spreading. At late times, some slow intermixing of the layers is exhibited. Though small differences in structure and spreading are apparent, the particles are remarkably close to each other considering the varying initial conditions.

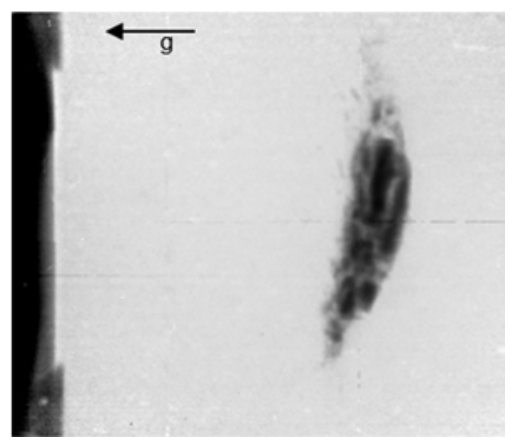

(a)

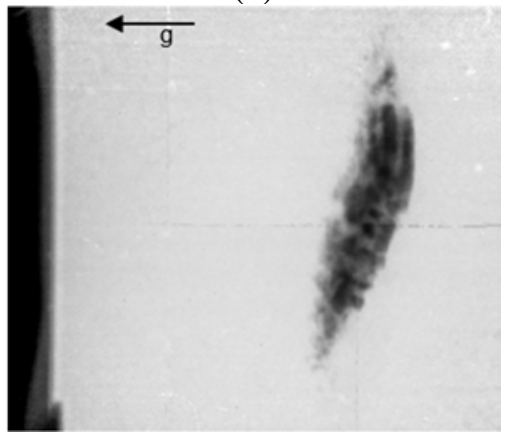

(c)

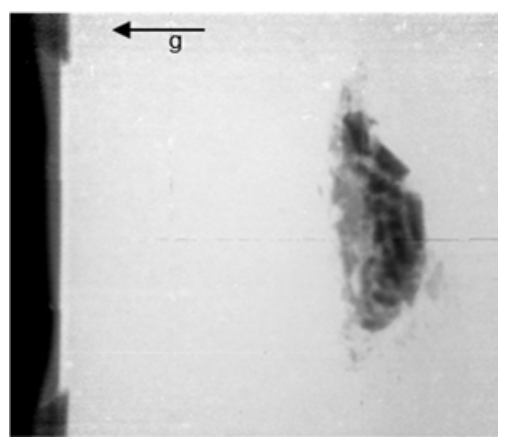

(b)

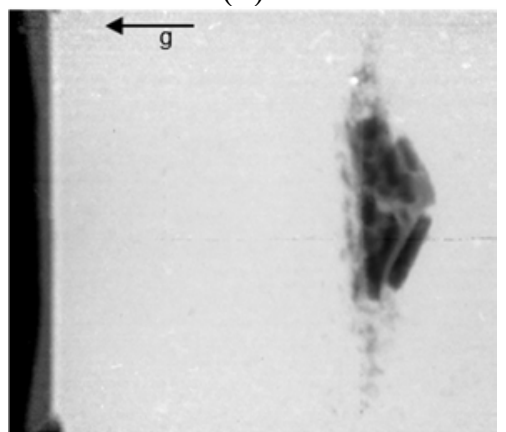

(d)

Figure 3: Transmission radiographs showing the dispersal of the particles $40 \mu$ s after detonator initiation for the four different test configurations: (a) Air; (b) Xenon; (c) SF6; (d) Vacuum. Flow is from left to right. 
Particle fronts are determined from thresholding of the transmission intensity. The intensity is filtered with a moving average with a 17 pixel window size. A background threshold is determined for each image line (see Fig. 4(a)). Casing location is determined from the first frame as the image location that drops below $40 \%$ of the background threshold. The $40 \%$ threshold was necessary to capture the location of the steel casing while ignoring the less dense aluminium retaining ring. In subsequent frames, the downstream front is determined as the image location where the intensity drops below $90 \%$ of the background and the upstream front is where the intensity rises again above the $90 \%$ threshold. Greater threshold values, such as $95 \%$ and $98 \%$, were attempted but produced too many spurious front locations. Sample particle front results are overlaid on the radiograph in Fig. 4(b). The seam down the centre of the image is a scintillator tile artefact. The reader will note that the seam is of sufficient contrast that it trips the particle front algorithm but is a spurious reading.

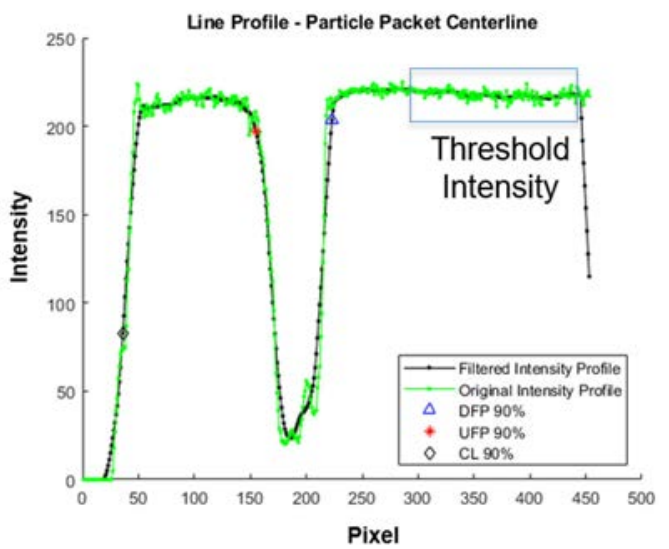

(a)

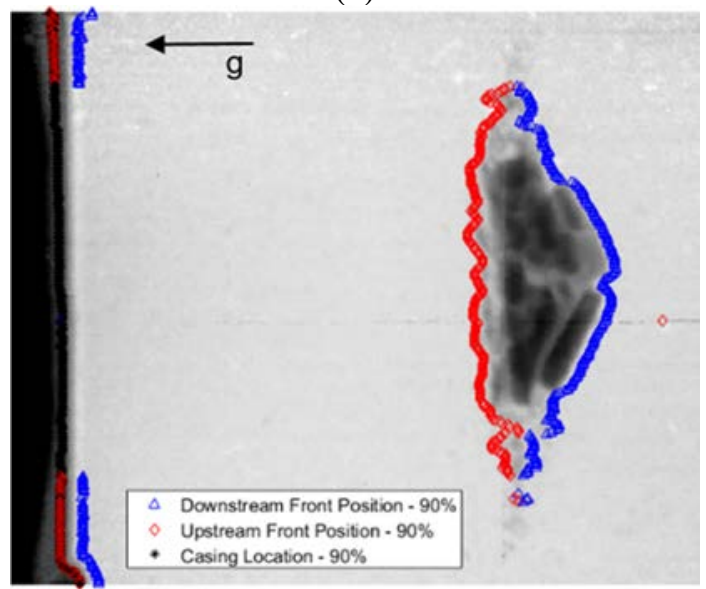

(b)

Figure 4: Threshold operations are used to identify the particle fronts. (a) Sample line profile showing the filtered intensity and the identification of the downstream front (blue triangle), upstream front (red star), and casing location (black diamond); (b) Results overlaid on the radiograph. Flow is from left to right. 
The behaviour of the particle packets at the centreline was extracted and plotted in Fig. 5. The initial time is from the detonation initiation. An approximately $4 \mu \mathrm{s}$ delay is present for the detonator to activate and for the explosive to completely react. The repeated air tests show close agreement on the centreline. One may note that the centreline results demonstrate a highly linear behaviour during this early time suggesting that the particles are impulsively acted upon by the contact interface and the shock. Furthermore, comparing the results of the four gas shots with the vacuum shot, we note that the results are within close agreement. The vacuum shot lacks an ambient medium for a shock to form, suggesting that the contact interface is almost solely responsible for the delivered impulse.

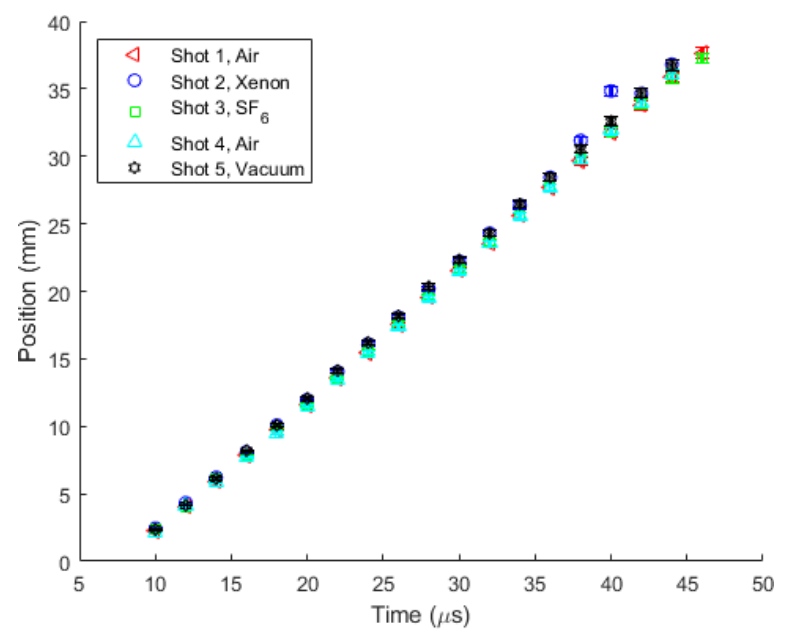

(a)

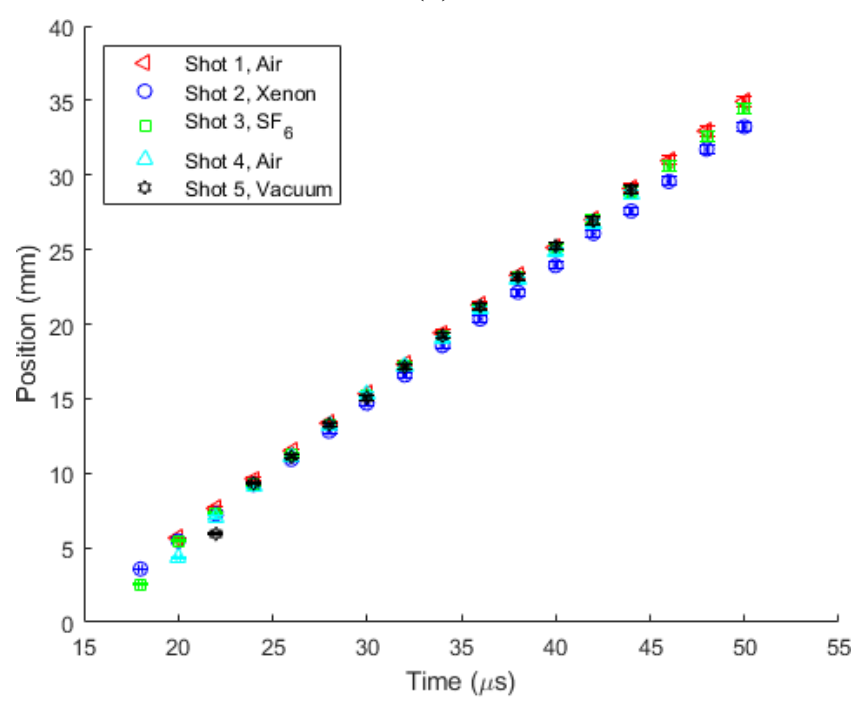

(b)

Figure 5: Centreline particle fronts obtained from the radiographs. (a) Downstream particle position; (b) Upstream particle position. 


\section{CONCLUSIONS}

A series of experiments performed at Los Alamos National Laboratory was aimed to provide validation data for numerical simulations performed at the Centre of Compressible Multiphase Turbulence at the University of Florida. The experiments were designed to be small scale, containing $\mathrm{O}\left(10^{5}\right)$ particles, to reduce the computational burden while maintaining the full physics of the explosive dispersal of particles. Five explosive tests were performed. A $13 \times 6 \mathrm{~mm}$ cylindrical packet of $100 \mu \mathrm{m}$ steel particles was dispersed by an explosive into three different carrier fluids: air, xenon, and $\mathrm{SF}_{6}$. A vacuum test was also performed to benchmark the relative strength of the shock compared to the contact interface. To penetrate the optically opaque explosive products at the early time and track the dispersed particle cloud, proton radiography was used. Twenty-one radiograph images at $2 \mu \mathrm{s}$ temporal resolution were obtained. However, as this study only presents the motion of the particle cloud, additional tests are needed to validate the accompanying particle-fluid interaction and explosive model. Tests of similar configuration, but that offer information on the behaviour of the shock and contact interface at early time, are needed to provide these necessary prediction metrics.

Centreline particle fronts for the three carrier phases demonstrate close agreement, suggesting the ambient carrier phase has little impact on the dispersal of the particles. Rather the forces exerted on the particle bed by the dense, propagating explosive products are dominant. Furthermore, the results demonstrate a highly linear behaviour during this early time suggesting that the particles are impulsively acted upon by the contact interface and the shock. Finally, comparing the results of the four gas shots with the vacuum shot, we note that the results are within close agreement. The vacuum shot lacks an ambient medium for a shock to form, suggesting that the contact interface is almost solely responsible for the delivered impulse.

\section{REFERENCES}

[1] Zhang, F., Frost, D.L., Thibault, P.A. \& Murray, S.B., Explosive dispersal of solid particles. Shock Waves, 10(6), pp. 431-443, 2001.

[2] Frost, D.L., Zarei, Z. \& Zhang, F., Instability of combustion products interface from detonation of heterogeneous explosives. International Colloquium on the Dynamics of Explosions and Reactive Systems, pp. 1-6, 2005.

[3] Frost, D.L., Ornthanalai, C., Zarei, Z., Tanguay, V. \& Zhang, F., Particle momentum effects from the detonation of heterogeneous explosives. Journal of Applied Physics, 101(11), p. 113529, 2007.

[4] Frost, D.L., Grégoire, Y., Petel, O., Goroshin, S. \& Zhang, F., Particle jet formation during explosive dispersal of solid particles. Physics of Fluids, 24(9), p. 091109, 2012.

[5] Sun, M., Saito, T., Takayama, K. \& Tanno, H., Unsteady drag on a sphere by shock wave loading. Shock Waves, 14(1-2), pp. 3-9, 2005.

[6] Tanno, H., Itoh, K., Saito, T., Abe, A. \& Takayama, K., Shock wave interaction with a sphere in a shock tube. Shock Waves, 13, pp. 483-497, 2004.

[7] Bredin, M.S. \& Skews, B.W., Drag measurement in unsteady compressible flow part 1: An unsteady flow facility and stress wave drag balance. $R \& D$ Journal of the South African Institution of Mechanical Engineering, 23(1), pp. 1-10, 2007.

[8] Maxey, M.R. \& Riley, J.J., Equation of motion for a small rigid sphere in a nonuniform flow. Physics of Fluids, 26(4) p. 883, 1983.

[9] Gatignol, R., The Faxén formulas for a rigid particle in an unsteady non-uniform Stokes-flow. Journal de Mécanique Théorique Appliquée, 2(2), pp. 143-160, 1983. 
[10] Parmar, M., Haselbacher, A. \& Balachandar, S., Generalized Basset-BoussinesqOseen equation for unsteady forces on a sphere in a compressible Flow. Physical Review Letters, 106(8), pp. 2-5, 2011.

[11] Parmar, M. \& Haselbacher, A., Balachandar, S., Equation of motion for a sphere in non-uniform compressible flows. Journal of Fluid Mechanics, 699(5), pp. 352-375, 2012.

[12] Annamalai, S. \& Balachandar, S., Faxén form of time-domain force on a sphere in unsteady spatially varying viscous compressible flows. Journal of Fluid Mechanics, 816, pp. 381-411, 2017.

[13] Britan, A., Elperin, T., Igra, O. \& Jiang, J.P., Acceleration of a sphere behind planar shock waves. Experiments in Fluids, 20(2), pp. 84-90, 1995.

[14] Jourdan, G., Houas, L., Igra, O., Estivalezes, J-L., Devals, C. \& Meshkov, E.E., Drag coefficient of a sphere in a non-stationary flow: New results. Proceedings of the Royal Society A: Mathematical, Physical and Engineering Sciences, 463(2088), pp. 33233245, 2007.

[15] Wagner, J.L., Beresh, S.J., Kearney, S.P., Pruett, B.O.M. \& Wright, E.K., Shock tube investigation of quasi-steady drag in shock-particle interactions. Physics of Fluids, 24(12), 123301, 2012.

[16] Bordoloi, A.D., Martinez, A.A. \& Prestridge, K., Relaxation drag history of shock accelerated microparticles. Journal of Fluid Mechanics, 823(R4), 2017.

[17] Hughes, K. et al., Uncertainty quantification of experiments on a small number of explosively-driven particles. 55th AIAA Aerospace Sciences, pp. 1-14, 2017.

[18] Rudinger, G., Some properties of shock relaxation in gas flows carrying small particles. Physics of Fluids, 7(5), pp. 658-663, 1964.

[19] Wagner, J.L. et al., A multiphase shock tube for shock wave interactions with dense particle fields. Experiments of Fluids, 52(6), pp. 1507-1517, 2012.

[20] Kellenberger, M., Johansen, C., Ciccarelli, G. \& Zhang, F., Dense particle cloud dispersion by a shock wave. Shock Waves, 23(5), pp. 415-430, 2013.

[21] Dullien, F.A.L., Porous Media : Fluid Transport and Pore Structure. 2nd ed., Elsevier Science, p. 598, 1992.

[22] King, N.S. et al., An 800-MeV proton radiography facility for dynamic experiments. Nuclear Instruments and Methods in Physics Research Section A: Accelerators, Spectrometers, Detectors and Associated Equipment, 424(1), pp. 84-91, 1999. 